\title{
Como incluir os cuidados paliativos na formação de médicos nefrologistas: Revisão
}

\section{da literatura}

\author{
How to include palliative care in training of nephrologist doctors: Literature review \\ Cómo incluir la atención paliativa en la formación de médicos nefrológos: Revisión de la literatura
}

Recebido: 21/04/2021 | Revisado: 30/04/2021 | Aceito: 05/05/2021 | Publicado: 19/05/2021

\author{
Ana Clara Aragão Fernandes \\ ORCID: https://orcid.org/0000-0002-8936-6599 \\ Universidade Potiguar, Brasil \\ E-mail: claraaragao2019@gmail.com \\ Jayana Teixeira Jales Menescal Pinto \\ ORCID: https://orcid.org/0000-0003-1450-5539 \\ Universidade Potiguar, Brasil \\ E-mail: jalesjayana@gmail.com \\ Larissa Câmara Nascimento de Melo \\ ORCID: https://orcid.org/0000-0001-5786-1096 \\ Universidade Potiguar, Brasil \\ E-mail: camaralarissa97@gmail.com \\ Juliana Ferreira Duarte \\ ORCID: https://orcid.org/0000-0002-1189-0876 \\ Universidade Potiguar, Brasil \\ E-mail: julianafd_@hotmail.com \\ Felipe Leite Guedes \\ ORCID: https://orcid.org/0000-0002-8348-0323 \\ Universidade Potiguar, Brasil \\ E-mail: felipeguedeshuol@gmail.com
}

\begin{abstract}
Resumo
Objetivo: $\mathrm{O}$ artigo tem como objetivo apresentar as vantagens dos cuidados paliativos $(\mathrm{CP})$ para pacientes com doença renal crônica (DRC) e evidenciar os conhecimentos necessários aos nefrologistas para oferecer um tratamento holístico e adequado a esses pacientes. Metodologia: Essa revisão foi produzida usando a Pubmed (Biblioteca Nacional Americana de Medicina), a Biblioteca Virtual em Saúde (BVS) e a Scientific Eletronic Library Online (SciELO). A busca avançada teve como descritores: cuidados paliativos, doença renal crônica e planejamento antecipado de cuidados. Posteriormente, filtrou-se os artigos em inglês e português com publicações entre 2015 e 2020, sendo selecionados os mais pertinentes. Resultados: A análise das referências elucidou o CP e o seu papel na DRC, as competências inerentes aos nefrologistas e os principais sintomas dos pacientes com DRC. Conclusão: A instituição do $\mathrm{CP}$, precocemente, é necessária para melhorar a qualidade de vida dos pacientes com DRC, sem restringir às medidas de fim de vida e sem excluir procedimentos intervencionistas. Assim, é indispensável investir na capacitação dos nefrologistas para que atendam às demandas da assistência paliativa em DRC.
\end{abstract}

Palavras-chave: Cuidados paliativos; Insuficiência renal crônica; Planejamento antecipado de cuidados; Nefrologia.

\begin{abstract}
Objective: The article aims to present the advantages of palliative care (PC) for patients with chronic kidney disease (CKD) and highlight the knowledge needed by nephrologists to offer holistic and appropriate treatment to these patients. Methods: This review was produced using Pubmed (American National Library of Medicine), the Virtual Health Library (VHL) and the Scientific Eletronic Library Online (SciELO). The advanced search had as descriptors: palliative care, chronic kidney disease and advance care planning. Subsequently, articles in English and Portuguese with publications between 2015 and 2020 were filtered, with the most relevant being selected. Results: The analysis of the references elucidated the PC and its role in CKD, the skills inherent to nephrologists and the main symptoms of patients with CKD. Conclusion: The early introduction of palliative care is necessary to improve the quality of life of patients with CKD, without restricting it to end-of-life measures and without excluding interventionist procedures. Thus, it is essential to invest in the training of nephrologists to meet the demands of palliative care in CKD.
\end{abstract}

Keywords: Palliative care; Chronic Renal insufficiency; Advance care planning; Nephrology.

\section{Resumen}

Objetivo: El artículo tiene como objetivo presentar las ventajas de los cuidados paliativos (CP) para los pacientes con insuficiencia renal crónica (IRC) y destacar los conocimientos que necesitan los nefrólogos para ofrecer un 
tratamiento holístico y adecuado a estos pacientes. Metodología: Esta revisión se produjo utilizando Pubmed (Biblioteca Nacional Estadounidense de Medicina), la Biblioteca Virtual en Salud (BVS) y la Biblioteca Científica Electrónica en Línea (SciELO). La búsqueda avanzada tuvo como descriptores: cuidados paliativos, enfermedad renal crónica y planificación anticipada de cuidados. Posteriormente, se filtraron los artículos en inglés y portugués con publicaciones entre 2015 y 2020, seleccionándose los más relevantes. Resultados: El análisis de las referencias esclareció la CP y su papel en la IRC, las habilidades inherentes a los nefrólogos y los principales síntomas de los pacientes con IRC. Conclusión: La institución de la CP, de manera temprana, es necesaria para mejorar la calidad de vida de los pacientes con IRC, sin restringirla a medidas de final de vida y excluyendo procedimientos intervencionistas. Por tanto, es fundamental invertir en la formación de nefrólogos para atender las demandas de los cuidados paliativos en la IRC.

Palabras clave: Cuidados paliativos; Insuficiencia renal crónica; Planificación anticipada de atención; Nefrología.

\section{Introdução}

De acordo com a Organização Mundial de Saúde, cuidados paliativos são incluídos em toda abordagem relacionada à melhora da qualidade de vida do paciente e de seus familiares. Este tratamento visa o alívio do sofrimento através da identificação e do tratamento precoce da dor e de outros problemas físicos, psíquicos, sociais, familiares e espirituais. São cuidados considerados imprescindíveis na abordagem de doenças crônicas que exigem atenção complexa durante a vida do paciente e objetiva a melhoria da qualidade de vida e a humanização do cuidado, atendendo às demandas prioritárias de cada indivíduo (ANCP, 2018).

Os princípios que determinam os cuidados paliativos, sob a atuação de uma equipe multidisciplinar, põem em foco a vida que o paciente ainda tem a viver e a tratativa da morte como processo natural. Em concordância, exige-se a integração de aspectos psicológicos e espirituais, além da participação ativa da família em toda a trajetória de doença, desde o diagnóstico até o fim da vida, inclusive durante o luto (Academia Nacional de Cuidados Paliativos, 2020). Isto posto, é indispensável dissociar a ideia de que essa modalidade de atendimento exclui indicação de procedimentos intervencionistas e diagnósticos, porém, é bem-estabelecido que todo e qualquer procedimento seja devidamente pactuado com o paciente, demonstrando claramente os riscos e benefícios daquilo que é proposto (WHO, 2016). Isto reforça o cuidado centrado no paciente, e não na doença, fazendo uso de planejamentos terapêuticos antecipados, haja vista a indispensabilidade de considerar os desejos do paciente, mesmo em situação de fim de vida (Scherer, Wright, Blaum \& Wall, 2017). Logo, a equipe multidisciplinar estabelece com o paciente o que se espera na progressão da doença, as perdas funcionais, o fardo dos sintomas e as dificuldades que permeiam sua vida doente, para que, munido de conhecimento, o paciente possa tomar decisões compartilhadas com seu médico a respeito do curso da enfermidade (Germain, 2015).

No que se refere a oferta desse recurso terapêutico, em 2017, foi realizado um mapeamento dos serviços especializados em cuidados paliativos, sendo evidenciado que os serviços existentes são insuficientes frente a demanda, não alcançando metade da população mundial. O Brasil situa-se na categoria relacionada aos países nos quais os serviços de cuidados paliativos estão em estágio preliminar de integração aos demais serviços de saúde (Clark et al., 2019).

Os cuidados paliativos são mais aceitos, praticados e acessíveis a pacientes portadores de neoplasias (Davison, Jhangri \& Koffman, 2014), mas a oferta de cuidados paliativos deve incluir pacientes portadores de doenças crônicas de diversas especialidades médicas, já que a progressão e a sintomatologia destas condições requerem uma complexa atenção. Correlacionando com ensaios clínicos randomizados realizados na Oncologia e Cardiologia, sugere-se que a adição dos cuidados paliativos ao tratamento padrão de condições clínicas de longo impacto, tais como a doenças da doença renal crônica (DRC), pode resultar em avanços substanciais na qualidade de vida, na diminuição dos sintomas, na depressão e ansiedade em comparação com o tratamento padrão (Quill \& Abernethy, 2013). Dessa forma, os cuidados paliativos devem ser inseridos na abordagem do paciente portador de DRC, dadas as características relacionadas à sua epidemiologia, gravidade, progressão e complexidade de seus sintomas e tratamentos relacionados. 
O objetivo deste estudo é apresentar conceitos relacionados aos cuidados paliativos em pacientes portadores de doença renal crônica, bem como enumerar as principais competências a serem desenvolvidas por médicos nefrologistas em formação.

\section{Metodologia}

Esta é uma revisão de literatura narrativa, utilizando as bases de dados eletrônicas Pubmed (Biblioteca Nacional Americana de Medicina), Biblioteca Virtual em Saúde (BVS) e Scientific Eletronic Library Online (SciELO). A pesquisa pelos estudos se deu no período entre 2015 - 2020, sendo buscados nos idiomas inglês e português e selecionados conforme os seguintes descritores de Ciências de Saúde: Cuidados paliativos, Doença renal crônica, e Planejamento Antecipado de Cuidados. Estes artigos foram filtrados por estudos em humanos e com foco em abordagens estatísticas. Nesta pesquisa, duplicatas e artigos que não se tratavam do tópico estudado foram excluídos. Não foram utilizados critérios baseados em Escala de Jadad, pontuação pela PEDro ou quality of research. Todos os artigos encontrados foram avaliados conjuntamente pelos cinco autores responsáveis.

\section{Resultados e Discussão}

A pesquisa nas bases de dados resultou em um total de 133 artigos por meio dos descritores citados, dos quais foram selecionados 42 artigos para análise. Destes, 16 estudos foram excluídos após leitura exploratória e seletiva. Assim, permaneceram 26 estudos para uso neste estudo após os critérios de inclusão e exclusão. Essa revisão explora diferentes tópicos como conceito de cuidados paliativos e o seu papel na doença renal crônica (DRC). A partir dos artigos selecionados foi possível contemplar os seguintes tópicos elementares: cuidados paliativos no contexto da DRC, competências necessárias para os médicos nefrologistas e principais sintomas apresentados por pacientes portadores de DRC.

\subsection{Cuidados paliativos no contexto da doença renal crônica: importância, início e inclusão na decisão do tratamento dialítico}

O conceito de suporte renal ("kidney supportive care") surge como uma subespecialidade crescente na Nefrologia, a qual integra a aplicação dos cuidados paliativos na Doença Renal Crônica, sobretudo quando em estágios avançados, independente de sua etiologia (Gelfand, Scherer \& Koncicki, 2020). Pacientes renais crônicos geralmente convivem com comorbidades que afetam outros sistemas orgânicos e que podem modificar a trajetória da doença (Bansal, Leonberg-Yoo, Schell, Scherer \& Jones, 2018). Algumas dessas condições interferem diretamente na saúde física, mental, no apoio familiar e social, que são importantes para a manutenção da qualidade de vida. Nesse caso, torna-se importante a inclusão do apoio familiar e de uma equipe multiprofissional aos cuidados, de maneira individualizada e contínua. (Gelfand, Scherer \& Koncicki, 2020; Davison, 2010).

Para que esse suporte seja realizado é necessário que a equipe multiprofissional de saúde tenha conhecimento sobre o que são os cuidados paliativos (Davison, 2010) e, assim, possam esclarecer e discutir com o paciente sobre as opções de manejo da sua doença. A falta de informação e de envolvimento dos pacientes nas decisões sobre o seu acompanhamento de saúde, acaba por interferir negativamente na adesão dos renais crônicos aos tratamentos recomendados. De acordo com um estudo canadense, que incluiu pacientes renais crônicos em estágios avançados (estágios 4 e 5), evidenciou que mais da metade do grupo foi submetido ao tratamento dialítico por decisão médica e cerca de $61 \%$ destes demonstraram algum arrependimento em realizar este procedimento, caso existissem outras estratégias de tratamento mais conservadoras.(Davison, 2010) Acredita-se que esse sentimento de arrependimento pode ser devido à depressão, transtorno prevalente em pacientes 
submetidos à diálise (Song, 2016). Um aspecto a ser considerado pelos profissionais da saúde é a preferência de doentes renais em fim de vida optarem por tratamento domiciliar ou em centros especializados em cuidados paliativos.

No público pediátrico, a inserção dos cuidados paliativos de forma precoce também traz benefícios no prognóstico e seguimento da DRC. Crianças submetidas à diálise e com comorbidades severas são mais hospitalizadas e têm aumento da morbidade (Neto, Moura \& Suassuna, 2017). Em crianças e adultos, os cuidados paliativos são promovidos tanto pela equipe multiprofissional - médicos, enfermeiros, psicólogos, técnicos de enfermagem e nutricionista - quanto pelos familiares e amigos. Esses cuidados vão desde avaliação e alívio dos sintomas, comunicação centrada no paciente, tomada de decisão compartilhada, avaliação do prognóstico regularmente até escolha do tratamento em conjunto com a finalidade de promover melhor qualidade de vida (Wachterman, Hailpern, Keating, Kurella Tamura, \& O'Hare, 2018). Diante disso, a escolha adequada do tratamento, planejamento futuro entre equipe multiprofissional, familiares e paciente e acompanhamento psicossocial são fundamentais para melhor manejo dessa condição de saúde.

A inclusão de modalidades terapêuticas individualizadas ao tratamento do paciente renal crônico que necessita de terapia renal substitutiva, seja por meio de peritoneal, hemodiálise domiciliar ou hemodiálise incremental tem se mostrado como alternativas ao tratamento dialítico convencional, e que pode ser inserido em contextos clínicos de pacientes que optam pela abordagem paliativa. A diálise peritoneal é realizada em ambiente domiciliar, requer treinamento e que o procedimento seja realizado pelo próprio paciente ou por um membro familiar capacitado, e sua avaliação da equipe multiprofissional é feita em consultas, no mínimo, mensais (Pirkle \& Junior, 2020). A hemodiálise domiciliar é uma modalidade subutilizada devido a barreiras orçamentárias, legais, educacionais e operacionais (Glickman \& Chan, 2021). Já no caso da hemodiálise incremental, que consiste na diálise menos frequente, realizada com o direcionamento de um objetivo específico (por exemplo, tratar a hiperpotassemia), carece resultados de estudos randomizados e controlados que demonstrem eficácia e sobrevida dos pacientes com essa terapia equiparado a pacientes que possuem o mesmo nível de função residual e que realizam a dose de hemodiálise completa (Bleyer \& Golper, 2019).

\subsection{Quais são as principais competências a serem apresentadas a nefrologistas em formação?}

Apesar das indicações e vantagens da aplicação dos cuidados paliativos, a maioria dos pacientes permanece sem nenhuma assistência paliativa, pois não há profissionais e serviços qualificados para fornecê-la (Academia Nacional de Cuidados Paliativos, 2020); Shah, Monga, Caperna \& Jhaveri, 2013). Em pesquisa nos Estados Unidos, com médicos nefrologistas foi mostrado que tópicos como "não oferecer tratamento agressivo", "retirada da hemodiálise" e "o que é cuidado paliativo" são desconfortáveis e muitas vezes negligenciados, sendo considerados difíceis de ser iniciados e preferivelmente não abordados (Shah et al., 2013). Ainda nessa pesquisa, 1 em cada 5 nefrologistas sentia-se obrigado a oferecer diálise independentemente de qual seja o prognóstico clínico do paciente (Shah et al., 2013). Dessa forma, a formação de nefrologistas requer capacitações em torno dos conhecimentos sobre os cuidados paliativos para o fornecimento das terapêuticas adequadas para suprir às reais necessidades dos pacientes.

A Kidney Disease: Improving Global Outcomes (KDIGO) estabelece cinco habilidades essenciais em assistência paliativa para nefrologistas exercerem o cuidado de suporte renal (Quadro 1): (a) identificação de pacientes para uso de cuidados conservativos de suporte, (b) pesquisa e manejo de sintomas, (c) comunicação de prognóstico, (d) habilidades de comunicação em decisão compartilhada e (e) uso dos serviços locais de cuidados paliativos (Quill \& Abernethy, 2013). A Matriz de Competências em Nefrologia, da Sociedade Brasileira de Nefrologia, também inclui cuidados paliativos como um dos pontos de formação do médico em seu último ano de formação em nefrologia (MEC, 2019).

A habilidade de identificação dos pacientes e de comunicação em decisão compartilhada resumem-se na abordagem em assistência paliativa, a qual deve ser iniciada antes da necessidade de ação ou ativação dos tratamentos de fim de vida. Esta 
deve ser iniciada na avaliação do paciente e suas comorbidades para determinação do nível de intensidade dos cuidados de suporte; além disso, engloba o diálogo sobre aspirações, metas de saúde e conceitos de qualidade de vida, visando atingir as dimensões não físicas de sofrimento que comprometem a vitalidade. (Quill \& Abernethy, 2013; Thumfart, Reindl, Rheinlaender, \& Müller, 2017). É importante ressaltar a necessidade de elucidação sobre cuidados paliativos para a remoção de estigmas criados em torno do termo, como relacionada apenas aos cuidados de fim de vida.

Tal abordagem precoce é importante, independente da faixa de idade, pois serve de amparo para todas as possibilidades de eventos adversos como infecções ou mortes abruptas. (Quill \& Abernethy, 2013; Thumfart et al., 2017) Pacientes, pais e familiares temem a morte prematura, e consideram importante a discussão detalhada da sua condição médica, possibilidades de tratamento e planos futuros em caso de morte (Davison, 2010; Thumfart et al., 2017), sendo importante que o nefrologista saiba manejar e conduzir esse tipo diálogo.

A tomada de decisão compartilhada deve analisar fatores como longevidade e os efeitos adversos diários da terapia, avaliando o custo-benefício do tratamento. Pacientes muito idosos e com múltiplas comorbidades tendem a ter expectativa de vida semelhante ao optar por tratamento conservador ou hemodialítico (Eckert, Motemaden \& Alves, 2018), com a desvantagem de sofrer longas hospitalizações e permanência ou aumento dos sintomas, caso optem pelo último. A terapia deve atender às reais expectativas e necessidades de saúde, devendo estes fatos serem avaliados e discutidos junto ao paciente para determinar a direção mais adequada do tratamento (Davison, 2010; Quill \& Abernethy, 2013).

O manejo dos principais sintomas é essencial, pois os pacientes com DRC geralmente sofrem de múltiplas comorbidades e possuem grande intensidade de sintomas, como dor e edema. A intervenção focada no manejo dos sintomas e com condutas centradas no paciente, focada nas dimensões não físicas de sofrimento, é associada a melhora física e emocional, além de melhora na qualidade de vida de pacientes em diálise, como discutido anteriormente (Siriwardana, Hoffman, Brennan, Li \& Brown, 2020). A avaliação da qualidade de vida é um ponto importante no manejo dos sintomas, entretanto, sua análise é subjetiva, pois é influenciada por fatores culturais e sociais, e sua avaliação deve sempre incluir: o status físico e mental, bem estar psicológico, presença de comorbidades e satisfação com a vida. (Eckert et al., 2018)

A característica da doença renal crônica como uma patologia com risco de vida é considerada abstrata e frequentemente negligenciada por familiares e profissionais, principalmente quando diagnosticada na infância. Entretanto, o entendimento do prognóstico pode influenciar na escolha do tratamento e no planejamento futuro do paciente. É observado que maioria dos pacientes deseja ter uma discussão franca sobre o seu prognóstico e possíveis condutas de terapias de fim de vida (Davison, 2010; Thumfart et al, 2017). Devendo o médico estar apto a realizar este diálogo e guiar o processo de tomada de decisão, em conjunto com o paciente, para o melhor tratamento individual. Como um passo inicial sugestiona-se elucidar como a doença pode afetar o cotidiano e quais sintomas podem ocorrer e implicar na qualidade de vida (Koncicki, \& Schell, 2016; Shah et al., 2013).

A utilização correta dos recursos locais de cuidados paliativos depende do nível de conhecimento dos profissionais locais sobre assistência paliativa e suas indicações, entretanto, o estigma e a falta de conhecimento sobre a temática privam o fornecimento desses serviços aos pacientes e, consequentemente, os afasta dos possíveis benefícios advindo dessa abordagem holística. Sendo assim, é de extrema a importância o mapeamento dos recursos e equipes locais e a capacitação profissional quanto a estas habilidades expostas pela KDIGO para realização destes cuidados, pois somente desta forma ocorrerá o referenciamento para as unidades especializadas nestes cuidados e o correto manejo do paciente (Quill \& Abernethy, 2013). 
Quadro 1 - Habilidades em cuidados paliativos para nefrologistas.

\begin{tabular}{|l|l|}
\hline \multicolumn{1}{|c|}{ Habilidades } & \multicolumn{1}{|c|}{ Descrição } \\
\hline $\begin{array}{l}\text { Identificação da indicação do uso de cuidados } \\
\text { conservativos de suporte para pacientes }\end{array}$ & $\begin{array}{l}\text { Avaliação do paciente, comorbidades, aspirações de vida, metas de saúde e conceitos } \\
\text { de qualidade de vida, visando determinar o nível de intensidade dos cuidados de } \\
\text { suporte. }\end{array}$ \\
\hline Pesquisa e manejo de sintomas & $\begin{array}{l}\text { Questionamento explícito da presença dos sintomas, recomenda-se o uso do IPOSs- } \\
\text { renal (Escala Integrada de Resultados de Cuidados Paliativos). }\end{array}$ \\
\hline Comunicação de prognóstico & $\begin{array}{l}\text { O manejo deve ser focado na avaliação da qualidade de vida. Questionar: o status } \\
\text { vida. }\end{array}$ \\
\hline Comunicação em decisão compartilhada bem estar psicológico, presença de comorbidades e satisfação com a \\
\hline Uso dos serviços locais de cuidados paliativos & $\begin{array}{l}\text { Esclarecimento sobre diagnóstico, implicações no cotidiano, surgimento de sintomas } \\
\text { e consequências para a qualidade de vida, visando auxiliar na escolha do tratamento } \\
\text { e planejamento futuro de vida. }\end{array}$ \\
\hline
\end{tabular}

Neste quadro estão as habilidades em cuidados paliativos necessárias ao nefrologista para a assistência adequada ao paciente. Fonte: Autores (2021).

\subsection{Abordagem clínica dos principais sintomas}

Pacientes com doença renal avançada apresentam carga pesada de sintomas físicos e psicológicos, (Gelfand, Scherer \& Koncicki, 2020) e a ocorrência desses sintomas é relatada entre os principais indicadores de redução da qualidade de vida relacionada a esse grupo (Siriwardana et al., 2020). Existe uma subnotificação dessa sintomatologia pelos pacientes, o que requer que sejam explicitamente questionados sobre sua ocorrência. Isso ocorre devido a associação de comorbidades existentes nesse grupo de doentes. Assim, sintomas importantes podem ser abordados de maneira inadequada pelo nefrologista, o qual pode direcionar sua atenção a uma série de marcadores bioquímicos e tratamentos direcionados ao procedimento de terapia renal substitutiva (Davison \& Jassal, 2016; Gelfand et al., 2020).

Em geral, a abordagem dos sintomas deve envolver a avaliação da causa, fatores reversíveis, nível de sofrimento ou disfunção causada, opções de intervenções não farmacológicas e farmacológicas, gerenciamento de expectativas e reconhecimento das limitações da terapia (Gelfand et al., 2020). Para facilitar a identificação e tratamento de sintomas em pacientes em diálise e/ou tratamento conservador recomenda-se o uso rotineiro de um questionário previamente estruturado, como o IPOSs-renal - Escala Integrada de Resultados de Cuidados Paliativos (Moskovitch, Mount \& Davies, 2019).

A versão renal da Escala Integrada de Resultados de Cuidados Paliativos (IPOS-renal) compreende a investigação de 17 sintomas físicos, sintomas emocionais e avaliação da comunicação e outros problemas práticos relacionados à DRC (Gelfand et al., 2020; Moskovitch et al., 2019). Estudos realizados na Austrália e Estados Unidos, entre os anos de 2015 e 2016, demonstram uma variação de 6 a 17 sintomas relatados na aplicação do questionário, porém esse número varia a depender das comorbidades associadas, da ferramenta de avaliação usada e do momento da medição (Moskovitch et al., 2019; Song, 2016).

Na prática do cuidado paliativo de suporte renal, muitas vezes os médicos precisam equilibrar o manejo de sintomas com o controle de comorbidades como: o controle ideal dos níveis de pressão arterial, anemia e fosfato; sem se concentrar na 
maximização da saúde a longo prazo, ou seja, na sobrevida do paciente, com ênfase ao apoio emocional, social e familiar. Para isso, muitos pacientes em hemodiálise ou em diálise peritoneal, necessitam da integração do suporte renal de qualidade para gerenciar os sintomas e otimizar a qualidade de vida. A abordagem dos sintomas é um dos principais objetivos do tratamento, visando a melhoria, pois nem sempre serão resolvidos completamente (Davison \& Jassal, 2016). Vários sintomas podem ser difíceis de ser manejados se tratados isoladamente, logo faz-se necessário avaliações frequentes e cuidadosas para atingir as metas do tratamento. A exemplo disso a adequação da diálise para sessões mais frequentes, porém mais curtas, pode ser mais aceitável para alguns pacientes, pois sintomas como distúrbios do sono, dificuldade respiratória e fadiga pós-diálise podem ser atenuados (Davison \& Jassal, 2016; Moskovitch et al., 2019).

A realização de hemodiálise, no entanto, pode não impactar na redução de sintomas experimentados (Song, 2016). Estudos de prevalência realizados no Reino Unido e Austrália, entre os anos de 2016 e 2017, descreveram a predominância de sintomas (fadiga ou falta de energia, baixa mobilidade, insônia, prurido, dor, dispneia e falta de apetite) em grupos de pacientes em hemodiálise ou em tratamento conservador. (Moskovitch et al., 2019; Siriwardana et al., 2020; Song, 2016). Do estudo em pacientes em diálise na Austrália em 2017, 53\% dos pacientes sentiu-se deprimido e 54\% sentiu-se ansioso (Siriwardana et al., 2020). No entanto, ocorreram diferenças significativas na prevalência de sintomas individuais. Pacientes em hemodiálise apresentaram dor com mais frequência e pacientes em diálise peritoneal apresentaram maiores taxas de sonolência e vômitos (Moskovitch et al., 2019). Embora tenha sido relatado uma prevalência semelhante de prurido entre os grupos, a intensidade no grupo de hemodiálise foi maior (Song, 2016). Cinco sintomas foram classificados como mais graves e com maior prevalência: insônia, dor, fadiga, falta de mobilidade e prurido (Siriwardana et al., 2020).

De modo geral a prática de exercícios físicos, terapia cognitivo-comportamental (TCC) e diálise mais frequente demonstram ser benéficos para distúrbios do sono, síndrome das pernas inquietas, depressão, dor e fadiga crônica (Davison \& Jassal, 2016; Gelfand et al., 2020). O uso de gabapentina em baixas doses (50 a 100mg pós-diálise) pode aliviar dores neuropáticas, prurido, pernas inquietas e distúrbios do sono, enquanto os antidepressivos podem melhorar o sono, apetite, fadiga e dor (Davison \& Jassal, 2016). Embora tenham demonstrado reduzir significativamente a intensidade do prurido nenhum medicamento foi aprovado pelo Food and Drug Administration para esta finalidade. Portanto é essencial a monitorização de sinais de toxicidade com ajuste cuidadoso da taxa de filtração glomerular (Gelfand et al., 2020). Estudos recentes sugerem que os pacientes em diálise que a pregabalina pode ser alternativa a não-respondedores à gabapentina (Mettang \& Kremer, 2015).

\section{Conclusão}

Os cuidados paliativos em pacientes com doença renal crônica devem ser instituídos precocemente no momento do diagnóstico da DRC, não ficando restritos a medidas de cuidados de fim de vida, e, além disso, é importante ressaltar que estes não excluem a realização de procedimentos intervencionistas e diagnósticos que alterariam o curso da doença. O suporte renal ("kidney supportive care") está relacionado a um conjunto de medidas que englobam o tratamento compartilhado centrado no paciente e sua família com planejamento terapêutico antecipado e adoção de condutas focadas na melhora da qualidade de vida, identificando e tratando problemas físicos e psíquicos e, assim, diminuindo o intenso fardo da doença crônica (ANCP, 2018; Quill \& Abernethy, 2013; Scherer et al., 2017; WHO, 2016).

Ao longo do estudo foram encontradas algumas limitações para a sua realização como a ausência de estudos analíticos que avaliem o benefício do tratamento conservador aliado aos cuidados paliativos frente às terapias de substituição renal. Além disso, pesquisas robustas acerca dos principais sintomas apresentados pelos pacientes com DRC e suas abordagem encontram-se em falta e desatualizadas, necessitando realizar novos estudos clínicos para melhor análise desta problemática. De forma semelhante, as pesquisas analisadas focavam, em sua maioria, nos pacientes, dificultando a avaliação 
completa no estudo do nefrologista frente aos pacientes com DRC - conduta, relação médico-paciente, terapia utilizada, abordagem dos sintomas.

Há crescente necessidade de capacitação dos nefrologistas e demais profissionais da saúde para atender as demandas de assistência paliativa em DRC, uma vez que nestes cenários há uma ideia obrigação em oferecer diálise independentemente de qual seja o prognóstico clínico e um grande desconforto em abordar tópicos como "não oferecer tratamento agressivo", "retirada da hemodiálise" e "o que é cuidado paliativo" (Shah et al., 2013). Dessa forma, é essencial que sejam desenvolvidas as habilidades clínicas em suporte renal como a identificação pacientes para uso de tratamento conservador, manejo dos sintomas, comunicação em decisão compartilhada e de prognóstico e, também, reconhecimento e uso dos serviços locais de cuidados paliativos como equipe multidisciplinar no cuidado ao paciente. Com estas habilidades bem instituídas, é possível estabelecer o tratamento direcionado às reais necessidades do paciente, excluindo terapias com intensos efeitos adversos que não modificam o curso da doença, fornecendo apoio familiar e abordagem holística para melhora da qualidade de vida.

Ao longo do desenvolvimento do presente estudo foram identificadas questões importantes que possibilitam a elaboração de novos estudos para melhor conhecimento e aplicação prática dos cuidados paliativos em pacientes com Doença Renal Crônica. Estudos analíticos que avaliassem a postura do nefrologista frente aos principais sintomas apresentados nos pacientes com DRC, com a finalidade analisar se as condutas estão promovendo uma melhor qualidade de vida desses pacientes seriam de suma importância para o manejo na pratica clínica diária dos nefrologistas.

\section{Referências}

Academia Nacional de Cuidados Paliativos. (2018). Análise situacional e recomendações para estruturação de programas de cuidados paliativos no Brasil. Ministério da Saúde. https://paliativo.org.br/wp-content/uploads/2018/12/ANALISE-SITUACIONAL_ANCP-18122018.pdf.

Bansal, A. D., Leonberg-Yoo, A., Schell, J. O., Scherer, J. S., \& Jones, C. A. (2018). Ten Tips Nephrologists Wish the Palliative Care Team Knew About Caring for Patients with Kidney Disease. Journal of palliative medicine, 21(4), 546-551. https://doi.org/10.1089/jpm.2018.0087

Bleyer, A., \& Golper, T. A. (2019). Incorporating residual kidney function into the dosing of intermittent hemodialysis. UpToDate. Retirado Setembro 19, 2019, de: https://www.uptodate.com/contents/incorporating-residual-kidney-function-into-the-dosing-of-intermittent-hemodialysis?source=history_widget

Brown, M. A., Collett, G. K., Josland, E. A., Foote, C., Li, Q., \& Brennan, F. P. (2015). CKD in Elderly Patients Managed without Dialysis: Survival, Symptoms, and Quality of Life. Clinical Journal of the American Society of Nephrology, 10(2), 260-268. 10.2215/cjn.03330414

Academia Nacional de Cuidados Paliativos (2020). O Manual de Cuidados Paliativos ANCP. Ministério da Saúde. http://biblioteca.cofen.gov.br/wpcontent/uploads/2017/05/Manual-de-cuidados-paliativos-ANCP.pdf.

Chettiar, A., Montez-Rath, M., Liu, S., Hall, Y. N., O'Hare, A. M., \& Kurella Tamura, M. (2018). Association of Inpatient Palliative Care with Health Care Utilization and Postdischarge Outcomes among Medicare Beneficiaries with End Stage Kidney Disease. Clinical journal of the American Society of Nephrology CJASN, 13(8), 1180-1187. https://doi.org/10.2215/CJN.00180118

Clark, D., Baur, N., Clelland, D., Garralda, E., López-Fidalgo, J., Connor, S., \& Centeno, C. (2019). Mapping levels of palliative care development in 198 countries: the situation in 2017. Journal of Pain and Symptom Management, 59, 794-807. 10.1016/j.jpainsymman.2019.11.009

Davison S. N. (2010). End-of-life care preferences and needs: perceptions of patients with chronic kidney disease. Clinical journal of the American Society of Nephrology: CJASN, 5(2), 195-204. https://doi.org/10.2215/CJN.05960809

Davison, S. N.,\& Jassal, S. V. (2016). Supportive Care: Integration of Patient-Centered Kidney Care to Manage Symptoms and Geriatric Syndromes. Clinical Journal of the American Society of Nephrology, 11(10), 1882-1891. 10.2215/cjn.01050116

Davison, S. N., Jhangri, G. S., \& Koffman, J. (2014). Knowledge of and attitudes towards palliative care and hospice services among patients with advanced chronic kidney disease. BMJ Supportive \& Palliative Care, 6(1), 66-74. 10.1136/bmjspcare-2013-000610

Davison, S. N., Koncicki, H., \& Brennan, F. (2014). Pain in Chronic Kidney Disease: A Scoping Review. Seminars in Dialysis, 27(2), 188-204. $10.1111 /$ sdi.12196.

Eckert, K., Motemaden, L., \& Alves, M. (2018). Effect of Hemodialysis Compared With Conservative Management on Quality of Life in Older Adults With End-Stage Renal Disease. Journal of Hospice \& Palliative Nursing, 20(3), 279-285. 10.1097/njh.0000000000000444.

Gelfand, S. L., Scherer, J. S., \& Koncicki, H. M. (2020). Kidney Supportive Care: Core Curriculum 2020. American Journal of Kidney Diseases, 75, 793-806. 10.1053/j.ajkd.2019.10.016.

Germain, M. J. (2015). How to Integrate Predictions in Outcomes in Planning Clinical Care. Blood Purification, 39, 65-69. 10.1159/000368946. 
Glickman, J. D., \& Chan, C. (2021). Home hemodialysis (HHD): Establishment of a program. UpToDate. https://www.uptodate.com/contents/incorporatingresidual-kidney-function-into-the-dosing-of-intermittent-hemodialysis?source=history_widget.

Koncicki, H. M., \& Schell, J. O. (2016). Communication Skills and Decision Making for Elderly Patients With Advanced Kidney Disease: A Guide for Nephrologists. American journal of kidney diseases: the official journal of the National Kidney Foundation,67(4), 688-695. https://doi.org/10.1053/j.ajkd.2015.09.032

Mettang, T., \& Kremer, A. E. (2015). Uremic pruritus. Kidney International, 87(4), 685-691. 10.1038/ki.2013.454

Ministério da Educação. (2019). Matriz de Competências em Nefrologia. http://portal.mec.gov.br/index.php?option=com_docman\&view=do wnload\&alias=128181- matriz-nefrologia-publ\&category_slug=novembro-2019\&Itemid=30192

Moskovitch, J. T., Mount, P. F., \& Davies, M. R. P. (2019). Changes in Symptom Burden in Dialysis Patients Assessed Using a Symptom-Reporting Questionnaire in Clinic. Journal of Palliative Care, 35, 59-65. 10.1177/0825859719827315

Neto, J. A. M., Moura, A. F. S., \& Suassuna, J. H. R. (2017). Renouncement of renal replacement therapy: withdrawal and refusal. Brazilian Journal of Nephrology, 39(3), 312-322. https://doi.org/10.5935/0101-2800.20170054

Pirkle, J. L., \& Junior, M.D. (2020). Prescribing peritoneal dialysis. UpToDate. https://www.uptodate.com/contents/prescribing-peritoneal- dialysis

Quill, T. E., \& Abernethy, A. P. (2013). Generalist plus Specialist Palliative Care - Creating a More Sustainable Model. New England Journal of Medicine, 368(13), 1173-1175. 10.1056/nejmp1215620.

Scherer, J. S., Wright, R., Blaum, C. S., \& Wall, S. P. (2017). Building an Outpatient Kidney Palliative Care Clinical Program. Journal of Pain and Symptom, 55, 108-116. 10.1016/j.jpainsymman.2017.08.005.

Shah, H. H., Monga, D., Caperna, A., \& Jhaveri, K. D. (2013). Palliative care experience of US adult nephrology fellows: a national survey. Renal Failure, 36(1), 39-45. 10.3109/0886022x.2013.831718

Siriwardana, A. N., Hoffman, A. T., Brennan, F. P., Li, K., \& Brown, M. (2020). A Impact of Renal Supportive Care on Symptom Burden in Dialysis Patients: A Prospective Observational Cohort Study. Journal of Pain and Symptom Management, 60, 725-736. 10.1016/j.jpainsymman.2020.04.030

Song, M. K. (2016). Quality of Life of Patients with Advanced Chronic Kidney Disease Receiving Conservative Care without Dialysis. Seminars in dialysis, 29(2), 165-169. https://doi.org/10.1111/sdi.12472 .

Thumfart, J., Reindl, T., Rheinlaender, C., \& Müller, D. (2017). Supportive palliative care should be integrated into routine care for paediatric patients with life-limiting kidney disease. Acta Paediatrica, 107(3), 403-407. 10.1111/apa.14182

Wachterman, M. W., Hailpern, S. M., Keating, N. L., Kurella Tamura, M., \& O'Hare, A. M. (2018). Association Between Hospice Length of Stay, Health Care Utilization, and Medicare Costs at the End of Life Among Patients Who Received Maintenance Hemodialysis. JAMA internal medicine, 178(6), 792-799. https://doi.org/10.1001/jamainternmed.2018.0256

World Health Organization (2016). Planning and implementing palliative care services: a guide for programme managers. World Health Organization. http://www.who.int/ncds/management/palliative-care/palliative_care_services/en/. 